Check for updates

Cite this: RSC Adv., 2017, 7, 46858

Received 5th September 2017

Accepted 27th September 2017

DOI: $10.1039 / c 7 r a 09858 h$

rsc.li/rsc-advances

\section{Design, synthesis, and herbicidal activity of pyrazole benzophenone derivatives $\uparrow$}

\author{
Ying Fu, (D) Meng-Xia Wang, Dong Zhang, Yu-Wen Hou, Shuang Gao, Li-Xia Zhao \\ and Fei Ye*
}

4-Hydroxyphenylpyruvate dioxygenase is one of the most promising targets for herbicide discovery. A series of 1-acyl-3-phenyl-pyrazol benzophenones were designed and synthesized using 1,3diphenylpropane-1,3-dione and dimethylformamide dimethylacetal as the starting materials. All of the compounds were characterized by $I R,{ }^{1} \mathrm{H} N M R,{ }^{13} \mathrm{C} N M R$, and HRMS. The configuration of $5 f$ was determined by X-ray crystallography. The bioassay studies indicated that most of these derivatives exhibited herbicidal activity at least to a certain degree, in which compounds $5 \mathrm{n}$ and 50 displayed good herbicidal activity at a dosage of $0.05 \mathrm{mmol} \mathrm{m}{ }^{-2}$, which were more potent than pyrazoxyfen against barnyard grass. In addition, compound 50 was also proved to be safer for maize than pyrazoxyfen. The binding free energy of compound 5o with HPPD was relatively low, and that agreed with the results of bioassay activity research. Therefore, compound 50 might be the lead compound for designing new HPPD inhibitors.

\section{Introduction}

4-Hydroxyphenylpyruvate dioxygenase (HPPD), a Fe(II)-dependent and non-heme dioxygenase, was founded by Zeneca Group PLC in 1982. ${ }^{1}$ HPPD catalyzes the conversion of 4-hydroxyphenylpyruvic acid (HPPA) to homogentisate (HGA) which involves decarboxylation, substituent migration and aromatic oxygenation in a single catalytic cycle..$^{2-4}$ HGA is an important precursor for the biosynthesis of tocopherol and plastoquinone, which are crucial for the normal growth of plants. The plant will be injured severely when HPPD is inhibited, and the plant meristem will become bleached in sunlight and cause necrosis and finally death..$^{5-7}$ Therefore, HPPD inhibitors are also termed bleaching herbicides. ${ }^{8,9}$ These herbicides have the advantages of a wide weed-control spectrum, flexibility for application time, and compatibility for tank mixes with other herbicides. ${ }^{10-13}$ Perennial broadleaf weeds in broad-leaf plant fields can also be weeded out by the HPPD inhibitor with high activities, lowresidue, and application safety. ${ }^{11,12,14}$ HPPD inhibitors have been developed a number of different structures, such as triketone, pyrazole, isoxazole, diketonitrile and benzophenone. ${ }^{15,16}$ As reported, containing triketone quinolines derivatives were designed and showed good herbicidal activity of Arabidopsis thaliana HPPD (AtHPPD). ${ }^{17}$ And Yang et al.

Department of Applied Chemistry, College of Science, Northeast Agricultural University, Harbin, 150030, P. R. China.E-mail: yefei@neau.edu.cn

$\dagger$ CCDC 1530820. For crystallographic data in CIF or other electronic format see DOI: $10.1039 / \mathrm{c} 7 \mathrm{ra09858h}$ developed a series of triketone-based hybrid compounds which lengthening the aryl side chain with potent inhibitory activity. ${ }^{18}$

The pyrazole and its derivatives have drawn wide attention in biological and pharmacological fields. ${ }^{19}$ As aromatic heterocyclic compounds, $N$-substituted pyrazole is important chemical scaffold, and a large number of pyrazole derivatives have been synthesized with extensive bioactivity. For example, $\mathrm{N}$ substituted pyrazoles are good inhibitors of p38 mitogenactivated protein kinase for the treatment of cancer cells. ${ }^{20}$ It was reported that the pyrazole-benzimidazolone is a potential inhibitor to type I tyrosinemia. ${ }^{21}$ Furthermore, pyrazolone-

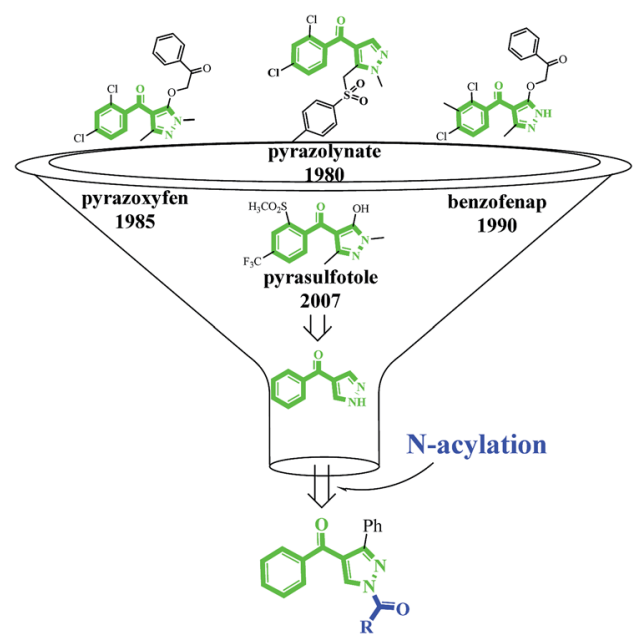

Fig. 1 Skeleton structure of the template compound. 


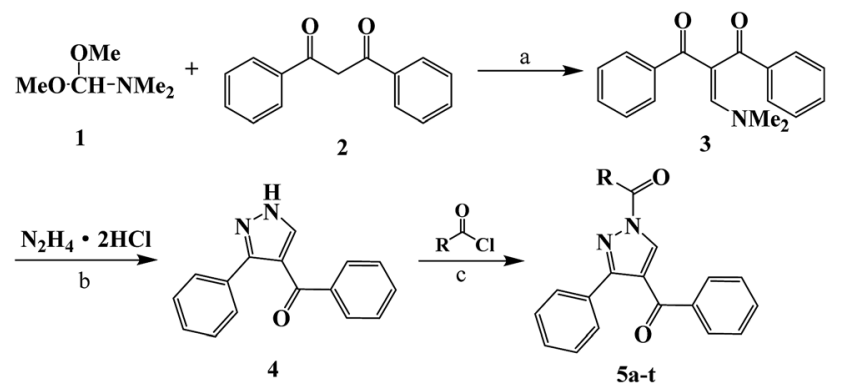

a: toluent, reflux 8h; b: ethanol, reflux $1.5 \mathrm{~h} ; \mathrm{c:} \mathrm{CH}_{2} \mathrm{Cl}_{2}$, reflux

Scheme 1 Route for synthesis of the target compounds.

quinazolone hybrids were confirmed as novel human 4hydroxyphenylpyruvate dioxygenase inhibitors. ${ }^{22}$

As shown in Fig. 1, there are four pyrazole HPPD inhibitors with good weed control containing pyrazolynate, pyrazoxyfen, benzofenap and pyrasulfotole. The common chemical subunit of these pyrazole inhibitors is 2-benzoylethen-1-ol. In order to search novel HPPD inhibitor analogues and continue to design and synthesis novel nitrogen-containing heterocycle pesticide, ${ }^{23-25}$ herein we reported the synthesis of a series of novel pyrazole derivatives via cyclization and acylation without any expensive reagent or catalyst (Scheme 1). Greenhouse experiments demonstrated that some of them exhibited promising herbicidal activity against barnyard grass at a rate of $0.05 \mathrm{mmol} \mathrm{m}^{-2}$.

\section{Experimental}

\section{Chemicals and instruments}

All the reagents were analytical grade and used without further purification. Analytical thin-layer chromatography (TLC) was performed on silica gel GF254 (Qingdao Haiyang Chemical Co. Ltd). The yields were not optimized. The melting point was measured using a Beijing Taike point apparatus (X-4) and the thermometer was not corrected. The infrared (IR) spectra were taken on a KJ-IN-27G infrared spectrophotometer $(\mathrm{KBr})$. The NMR spectra were recorded on a Bruker AV600 spectrometer with $\mathrm{CDCl}_{3}$ or DMSO- $\mathrm{d}_{6}$ as the solvent and TMS as the internal standard. High-resolution mass spectrometry (HRMS) was obtained by FTICR-MS. Pyrazoxyfen were purchased from Tianjin Sigma Technology Co., Ltd.

\section{Synthesis of 2-dimethylaminomethylene-1,3-} diphenylpropane-1,3-dione (3)

Compound 3 was synthesized according to the ref. 26. A mixture of 1,3-diphenylpropane-1,3-dione $(2.24 \mathrm{~g}, 10 \mathrm{mmol})$ and dimethylformamide dimethylacetal $(1.19 \mathrm{~g}, 10 \mathrm{mmol})$ in anhydrous toluene $(50 \mathrm{~mL})$ was heated and refluxed for $8 \mathrm{~h}$, then cooled to room temperature. The compound 3 was obtained as faint yellow solid in $44 \%$ yield. Mp: $131-132{ }^{\circ} \mathrm{C} ;{ }^{1} \mathrm{H}$ NMR $(600 \mathrm{MHz}$, $\left.\mathrm{CDCl}_{3}\right)$ : 2.83-3.14, $\left(6 \mathrm{H},-\mathrm{CH}_{3}\right) ; 7.59-7.16,(\mathrm{~m}, 10 \mathrm{H}, \mathrm{Ar}-\mathrm{H}) ; 7.64$, $(\mathrm{s}, 1 \mathrm{H}, \mathrm{N}-\mathrm{CH}=\mathrm{C}) ;{ }^{13} \mathrm{C}$ NMR $\left(150 \mathrm{MHz}, \mathrm{CDCl}_{3}\right): 42.3,47.3,111.5$, 127.9 (4C), 129.0 (4C), 131.2 (2C), 141.0 (2C), 158.1, 194.9 (2C); IR ( $\left.\mathrm{KBr}, \mathrm{cm}^{-1}\right): 3051,2923,1651,1584,1562$.
Synthesis of phenyl-(3-phenyl-1H-pyrazol-4-yl) methanone (4)

Synthesis of compound $\mathbf{4}$ was based on the method presented earlier. ${ }^{26-29}$ Compound $3(2.7 \mathrm{~g}, 10 \mathrm{mmol})$ was treated with $\mathrm{N}_{2} \mathrm{H}_{4} \cdot 2 \mathrm{HCl}$ (1.04 g, $10 \mathrm{mmol}$ ) and refluxed in ethanol for $1.5 \mathrm{~h}$, and appropriate distilled water was added to the solution before heating. The faint yellow solid product was obtained, filtered and recrystallized from ethanol. Compound 4 was obtained with the yield of $90 \%$. Mp: $165-166{ }^{\circ} \mathrm{C} ;{ }^{1} \mathrm{H}$ NMR $(600 \mathrm{MHz}$, DMSO-d $\left._{6}\right)$ : 7.38-7.78, $(\mathrm{m}, 10 \mathrm{H}, \mathrm{Ar}-\mathrm{H}) ; 8.10,(\mathrm{~s}, 1 \mathrm{H}, \mathrm{N}-\mathrm{CH}=\mathrm{C})$; 13.63, (br, $1 \mathrm{H}, \mathrm{NH}) ;{ }^{13} \mathrm{C}$ NMR (150 MHz, DMSO-d $\left.{ }_{6}\right): 118.3,128.6$, 128.9 (2C), 129.1 (3C), 129.5 (4C), 132.8 (3C), 139.4, 190.1; IR $\left(\mathrm{KBr}, \mathrm{cm}^{-1}\right): 3345,1651,1562,1433$.

\section{General synthetic procedures for compounds 5a-t}

Acyl chloride $(12 \mathrm{mmol})$ were added dropwise to a solution of compound 4 ( $2.48 \mathrm{~g}, 10 \mathrm{mmol})$, and triethylamine (1.52 g, 15 $\mathrm{mmol}$ ) as the attaching acid agent in $\mathrm{CH}_{2} \mathrm{Cl}_{2}$, and the mixture was refluxed for $4 \mathrm{~h}$ until the reaction was complete (indicated by TLC). The organic phase was washed with water until $\mathrm{pH}=7$. The organic layer was dried over anhydrous $\mathrm{MgSO}_{4}$ and vacuum distillation solvent. Compounds $\mathbf{5 a - t}$ was obtained by recrystallized with $\mathrm{CH}_{2} \mathrm{Cl}_{2}$ and light petroleum or column chromatography with $\mathrm{CH}_{2} \mathrm{Cl}_{2}$ and EtOH. The physical and spectra data of compounds $\mathbf{5 a - t}$ were as follows:

3-Phenyl-1-benzoylpyrazole-4-benzophenone (5a). White solid; yield: $87 \%$; mp: $165-167{ }^{\circ} \mathrm{C} ;{ }^{1} \mathrm{H}$ NMR (600 MHz, $\mathrm{CDCl}_{3}$ ): 7.38-8.33, (m, 15H, Ar-H); 8.75, (s, $1 \mathrm{H}, \mathrm{N}-\mathrm{CH}=\mathrm{C}) ;{ }^{13} \mathrm{C}$ NMR (150 MHz, $\mathrm{CDCl}_{3}$ ): 122.4, 128.3 (2C), 28.4, 128.7 (2C), 128.9 (2C), 129.4 (2C), 129.7 (2C), 130.6, 131.1, 132.1 (2C), 133.4, 133.7, 135.4, 138.1, 155.8, 165.9, 189.8; IR $\left(\mathrm{KBr}, \mathrm{cm}^{-1}\right): 3154,3062$, 1706, 1654, 1596, 1446; HRMS (ESI): $m / z\left[\mathrm{M}+\mathrm{H}^{+}\right]$calculated for monoisotopic mass 353.1212, found 353.1286.

3-Phenyl-1-o-methylbenzoyl-pyrazole-4-benzophenone (5b). White solid; yield: 90\%; mp: $150-152{ }^{\circ} \mathrm{C} ;{ }^{1} \mathrm{H}$ NMR (600 MHz, $\left.\mathrm{CDCl}_{3}\right): 2.48,\left(\mathrm{~s}, 3 \mathrm{H},-\mathrm{CH}_{3}\right) ; 7.26-7.93,(\mathrm{~m}, 14 \mathrm{H}, \mathrm{Ar}-\mathrm{H}) ; 8.66$, (s, $1 \mathrm{H}, \mathrm{N}-\mathrm{CH}=\mathrm{C}) ;{ }^{13} \mathrm{C} \mathrm{NMR}\left(150 \mathrm{MHz}, \mathrm{CDCl}_{3}\right): 20.3,122.7,125.3$, 128.3 (2C), 128.7 (2C), 128.9 (2C), 129.4, 129.7 (2C), 130.3, 131.0, 131.1, 131.3, 131.9, 133.5, 134.6, 138.0, 138.4, 155.9, 167.6, 189.8; IR (KBr, $\left.\mathrm{cm}^{-1}\right): 3124,3031,1713,1655,1599,1449$; HRMS (ESI): $m / z\left[\mathrm{M}+\mathrm{H}^{+}\right]$calculated for monoisotopic mass 367.1368 , found 367.1443.

3-Phenyl-1-m-methylbenzoyl-pyrazole-4-benzophenone (5c). Faint yellow solid; yield: $86 \%$; mp: $155-156{ }^{\circ} \mathrm{C} ;{ }^{1} \mathrm{H}$ NMR $(600$ $\left.\mathrm{MHz}, \mathrm{CDCl}_{3}\right): 2.47,\left(\mathrm{~s}, 3 \mathrm{H},-\mathrm{CH}_{3}\right) ; 7.37-8.13,(\mathrm{~m}, 14 \mathrm{H}, \mathrm{Ar}-\mathrm{H})$; 8.73, (s, $1 \mathrm{H}, \mathrm{N}-\mathrm{CH}=\mathrm{C}) ;{ }^{13} \mathrm{C}$ NMR $\left(150 \mathrm{MHz}, \mathrm{CDCl}_{3}\right): 21.5,122.3$, 128.2, 128.4 (2C), 128.7 (2C), 128.9 (2C), 129.3, 129.4, 129.7 (2C), 130.5, 131.2, 132.4, 133.4, 134.6, 135.5, 138.1, 138.2, 155.7, 166.2, 189.8; IR $\left(\mathrm{KBr}, \mathrm{cm}^{-1}\right): 3153,3060,1688,1662,1597,1447$; HRMS (ESI): $m / z\left[\mathrm{M}+\mathrm{H}^{+}\right]$calculated for monoisotopic mass 367.1368 , found 367.1444 .

3-Phenyl-1-p-methylbenzoyl-pyrazole-4-benzophenone (5d). White solid; yield: $89 \%$; mp: $175-177{ }^{\circ} \mathrm{C} ;{ }^{1} \mathrm{H}$ NMR $(600 \mathrm{MHz}$, $\left.\mathrm{CDCl}_{3}\right): 2.45,\left(\mathrm{~s}, 3 \mathrm{H},-\mathrm{CH}_{3}\right) ; 7.24-8.23,(\mathrm{~m}, 14 \mathrm{H}, \mathrm{Ar}-\mathrm{H}) ; 8.71(\mathrm{~s}$, $1 \mathrm{H}, \mathrm{N}-\mathrm{CH}=\mathrm{C}) ;{ }^{13} \mathrm{C}$ NMR $\left(150 \mathrm{MHz}, \mathrm{CDCl}_{3}\right): 21.8,122.1,127.7$, 128.3, 128.4, 128.6 (2C), 128.9, 129.1 (2C), 129.3, 129.6 (2C), $131.2,132.1,132.3,132.5,133.3,135.4,138.2,144.8,155.6$, 
165.7, 189.8; IR (KBr, $\left.\mathrm{cm}^{-1}\right): 3154,3032,1698,1654,1595,1444$; HRMS (ESI): $m / z\left[\mathrm{M}+\mathrm{H}^{+}\right]$calculated for monoisotopic mass 367.1368, found 367.1444.

3-Phenyl-1-o-chlorinebenzoyl-pyrazole-4-benzophenone (5e). White solid; yield: $83 \%$; mp: $140-142{ }^{\circ} \mathrm{C} ;{ }^{1} \mathrm{H}$ NMR $(600 \mathrm{MHz}$, $\left.\mathrm{CDCl}_{3}\right)$ : 7.29-7.90, (m, $\left.14 \mathrm{H}, \mathrm{Ar}-\mathrm{H}\right) ; 8.64,(\mathrm{~s}, 1 \mathrm{H}, \mathrm{N}-\mathrm{CH}=\mathrm{C}) ;{ }^{13} \mathrm{C}$ NMR (150 MHz, $\mathrm{CDCl}_{3}$ ): 123.2, 126.6, 128.3 (2C), 128.7 (2C), 128.9 (2C), 129.4, 129.6 (2C), 130.1, 130.4, 130.8, 132.2, 132.5, 132.6, 133.5, 133.9, 137.9, 156.2, 165.6, 189.6; IR (KBr, $\left.\mathrm{cm}^{-1}\right)$ : 3126, 3032, 1735, 1662, 1598, 1448; HRMS (ESI): $m / z\left[\mathrm{M}+\mathrm{H}^{+}\right]$ calculated for monoisotopic mass 387.0822, found 387.0895.

3-Phenyl-1-p-chlorinebenzoyl-pyrazole-4-benzophenone (5f). Faint yellow solid; yield: $80 \%$; mp: $183-184{ }^{\circ} \mathrm{C} ;{ }^{1} \mathrm{H}$ NMR $(600$ $\left.\mathrm{MHz}, \mathrm{CDCl}_{3}\right)$ : 7.37-8.31, (m, 14H, Ar-H); 8.74, (s, 1H, N-CH= C); ${ }^{13} \mathrm{C}$ NMR (150 MHz, $\mathrm{CDCl}_{3}$ ): 122.5, 128.4 (2C), 128.7 (4C), 128.8 (2C), 128.9, 129.5, 129.7 (2C), 130.9, 133.5, 133.6 (2C), 135.3, 138.0, 140.4, 156.0, 164.9, 189.7; IR $\left(\mathrm{KBr}, \mathrm{cm}^{-1}\right): 3145$, 3036, 1707, 1659, 1589, 1447; HRMS (ESI): $m / z\left[\mathrm{M}+\mathrm{H}^{+}\right]$calculated for monoisotopic mass 387.0822, found 387.0896.

3-Phenyl-1-p-fluorobenzoyl-pyrazole-4-benzophenone (5g). White solid; yield: $79 \%$; mp: $174-176{ }^{\circ} \mathrm{C} ;{ }^{1} \mathrm{H}$ NMR $(600 \mathrm{MHz}$, $\left.\mathrm{CDCl}_{3}\right): 7.21-8.45,(\mathrm{~m}, 14 \mathrm{H}, \mathrm{Ar}-\mathrm{H}) ; 8.75,(\mathrm{~s}, 1 \mathrm{H}, \mathrm{N}-\mathrm{CH}=\mathrm{C}) ;{ }^{13} \mathrm{C}$ NMR (150 MHz, $\mathrm{CDCl}_{3}$ ): 115.5, 115.8, 122.3, 126.7, 128.4 (2C), 128.7 (2C), 128.8 (2C), 129.5 (2C), 129.7 (2C), 131.0, 133.5, 135.0, 135.2, 135.4, 138.0, 155.9, 164.6, 189.7; IR $\left(\mathrm{KBr}, \mathrm{cm}^{-1}\right): 3152$, 3059, 1692, 1668, 1598, 1448; HRMS (ESI): $m / z\left[\mathrm{M}+\mathrm{H}^{+}\right]$calculated for monoisotopic mass 371.1118, found 371.1193.

3-Phenyl-1-p-trifluoromethylbenzoyl-pyrazole-4-benzophenone (5h). White solid; yield: 78\%; mp: $173-175{ }^{\circ} \mathrm{C} ;{ }^{1} \mathrm{H}$ NMR $(600 \mathrm{MHz}$, $\left.\mathrm{CDCl}_{3}\right)$ : 7.38-8.43, (m, 14H, Ar-H); 8.77, (s, $\left.1 \mathrm{H}, \mathrm{N}-\mathrm{CH}=\mathrm{C}\right) ;{ }^{13} \mathrm{C}$ NMR (150 MHz, $\left.\mathrm{CDCl}_{3}\right)$ : 122.9, 125.2, 125.3, 128.5 (2C), 128.7 (2C), 128.8 (2C), 129.0, 129.6 (2C), 129.7 (2C), 130.8, 132.4 (2C), 133.6, 133.9, 134.6, 135.1, 137.9, 156.2, 164.9, 189.6; IR (KBr, cm$\left.{ }^{-1}\right)$ : 3175, 3061, 1715, 1664, 1597, 1446; HRMS (ESI): $m / z\left[\mathrm{M}+\mathrm{H}^{+}\right]$ calculated for monoisotopic mass 421.1086 , found 421.1159 .

3-Phenyl-1-p-nitrobenzoyl-pyrazole-4-benzophenone

(5i).

Grey white powder; yield: $30 \%$; mp: $79-81{ }^{\circ} \mathrm{C} ;{ }^{1} \mathrm{H}$ NMR $(600$ $\left.\mathrm{MHz}, \mathrm{CDCl}_{3}\right)$ : 7.38-8.49, (m, 14H, Ar-H); 8.76, (s, $1 \mathrm{H}, \mathrm{N}-\mathrm{CH}=$ C); ${ }^{13} \mathrm{C}$ NMR (150 MHz, $\mathrm{CDCl}_{3}$ ): 123.2, 123.3 (2C), 128.5 (5C), 129.7 (2C), 129.7 (2C), 130.5, 133.0 (2C), 133.6, 134.8, 136.1, 137.8, 150.5, 156.5, 164.3, 189.5; IR (KBr, $\left.\mathrm{cm}^{-1}\right)$ : 3134, 3051, 1715, 1657, 1596, 1447; HRMS (ESI): $m / z\left[\mathrm{M}+\mathrm{H}^{+}\right]$calculated for monoisotopic mass 398.1063, found 398.1129.

3-Phenyl-1-(2', $\mathbf{4}^{\prime}$-dichlorine)benzoyl-pyrazole-4-benzophenone (5j). White solid; yield: $81 \%$; mp: $207-209{ }^{\circ} \mathrm{C} ;{ }^{1} \mathrm{H}$ NMR $(600 \mathrm{MHz}$, $\left.\mathrm{CDCl}_{3}\right)$ : 7.31-7.90, (m, 13H, Ar-H); 8.65, (s, $\left.1 \mathrm{H}, \mathrm{N}-\mathrm{CH}=\mathrm{C}\right) ;{ }^{13} \mathrm{C}$ NMR (150 MHz, $\mathrm{CDCl}_{3}$ ): 123.3, 127.0, 128.3 (2C), 128.7 (2C), 128.8 (2C), 129.6 (3C), 130.2, 130.5, 130.6, 131.4, 133.6, 133.7, 133.8, 137.8, 138.3, 156.3, 164.8, 189.49; IR (KBr, $\left.\mathrm{cm}^{-1}\right): 3148 ; 3056$, 1727, 1660, 1581, 1447; HRMS (ESI): $m / z\left[\mathrm{M}+\mathrm{H}^{+}\right]$calculated for monoisotopic mass 421.0432, found 421.0505.

3-Phenyl-1-(3'-hydroxy-4'-chlorine)benzoyl-pyrazole-4-benzophenone (5k). Grey white powder; yield: $29 \%$; mp: $219-221{ }^{\circ} \mathrm{C}$; ${ }^{1} \mathrm{H}$ NMR (600 MHz, DMSO-d 6 ): 7.39-7.93, (m, 13H, Ar-H); 8.84, $(\mathrm{s}, 1 \mathrm{H}, \mathrm{N}-\mathrm{CH}=\mathrm{C}) ; 10.86(\mathrm{~s}, 1 \mathrm{H},-\mathrm{OH}) ;{ }^{13} \mathrm{C} \mathrm{NMR}(150 \mathrm{MHz}$, DMSO-d ${ }_{6}$ ): 119.7, 122.0, 123.7, 126.1, 128.8 (2C), 129.0 (2C), 129.2 (2C), 129.7, 123.0 (2C), 130.4, 130.5, 131.4, 134.1, 136.2,
138.0, 153.3, 155.0, 165.0, 189.8; IR (KBr, $\left.\mathrm{cm}^{-1}\right)$ : 3272, 3145, 3065, 1710, 1638, 1590, 1415; HRMS (ESI): $m / z\left[\mathrm{M}+\mathrm{H}^{+}\right]$calculated for monoisotopic mass 403.0771, found 403.0844 .

3-Phenyl-1-(2'-chlorine-4'-methylsulfonyl)benzoyl-pyrazole4-benzophenone (5l). White solid; yield: 73\%; mp: $216-218{ }^{\circ} \mathrm{C}$; ${ }^{1} \mathrm{H}$ NMR (600 MHz, $\left.\mathrm{CDCl}_{3}\right): 3.12,\left(\mathrm{~s}, 3 \mathrm{H},-\mathrm{CH}_{3}\right) ; 7.30-8.10,(\mathrm{~m}$, $13 \mathrm{H}, \mathrm{Ar}-\mathrm{H}) ; 8.69,(\mathrm{~s}, 1 \mathrm{H}, \mathrm{N}-\mathrm{CH}=\mathrm{C}) ;{ }^{13} \mathrm{C} \mathrm{NMR}\left(150 \mathrm{MHz}, \mathrm{CDCl}_{3}\right)$ : 44.4, 123.7, 125.6, 128.4 (2C), 128.8 (5C), 129.0, 129.7 (2C), $129.8,130.3$, 131.0, 133.4, 133.7, 137.4, 137.6, 144.1, 156.8, 164.3, 189.3; IR (KBr, cm ${ }^{-1}$ ): 3106, 2928, 1732, 1654, 1598, 1448; HRMS (ESI): $m / z\left[\mathrm{M}+\mathrm{H}^{+}\right]$calculated for monoisotopic mass 465.0598, found 465.0672 .

3-Phenyl-1-(2'-nitro-4' -methylsulfonyl)benzoyl-pyrazole-4benzophenone $(\mathbf{5 m})$. White solid; yield: $71 \%$; mp: $272-$ $274{ }^{\circ} \mathrm{C} ;{ }^{1} \mathrm{H}$ NMR $\left(600 \mathrm{MHz}, \mathrm{CDCl}_{3}\right): 3.21$, (s, 3H, $\left.-\mathrm{CH}_{3}\right) ; 7.28-$ 8.72, (m, 13H, Ar-H); 8.82, (s, $1 \mathrm{H}, \mathrm{N}-\mathrm{CH}=\mathrm{C}) ;{ }^{13} \mathrm{C}$ NMR $(150$ $\mathrm{MHz} \mathrm{CDCl}_{3}$ ): 44.4, 123.5, 123.8, 128.4 (2C), 128.7 (2C), 128.8 (2C), 129.6 (2C), 129.8, 130.1, 131.4, 132.7, 132.9, 133.5, $133.7,137.6,144.55,147.9,156.9,163.6,189.2$; IR $\left(\mathrm{KBr}, \mathrm{cm}^{-1}\right): 3135,2923,1727,1655,1596,1450$; HRMS (ESI): $\mathrm{m} / \mathrm{z}\left[\mathrm{M}+\mathrm{H}^{+}\right]$calculated for monoisotopic mass 476.0838, found 476.0911 .

3-Phenyl-1-furoyl-pyrazole-4-benzophenone (5n). Grey white powder; yield: $73 \%$; mp: $177-179{ }^{\circ} \mathrm{C} ;{ }^{1} \mathrm{H} \mathrm{NMR}$ (600 $\mathrm{MHz}, \mathrm{CDCl}_{3}$ ): 6.67-8.27, (m, 13H, Ar-H, O-CH= $\mathrm{CH}-\mathrm{CH}=)$; 8.72, (s, $1 \mathrm{H}, \mathrm{N}-$ $\mathrm{CH}=\mathrm{C}) ;{ }^{13} \mathrm{C} \mathrm{NMR}\left(150 \mathrm{MHz}, \mathrm{CDCl}_{3}\right): 113.0,122.2,126.0,128.4$ (2C), 128.7 (2C), 128.8 (2C), 129.5, 129.6 (2C), 131.1, 133.4, 134.8, 138.1, 144.3, 149.0, 154.4, 156.1, 189.5; IR (KBr, $\left.\mathrm{cm}^{-1}\right)$ : 3155, 3066, 1696, 1652, 1595, 1445; HRMS (ESI): $m / z\left[\mathrm{M}+\mathrm{H}^{+}\right]$ calculated for monoisotopic mass 343.1004, found 343.1077.

3-Phenyl-1-phenoxyacetyl-pyrazole-4-benzophenone (5o). White solid; yield: 76\%; mp: $128-130{ }^{\circ} \mathrm{C}$; ${ }^{1} \mathrm{H}$ NMR $(600 \mathrm{MHz}$, $\left.\mathrm{CDCl}_{3}\right)$ : 5.62, (s, $\left.2 \mathrm{H},-\mathrm{CH}_{2}-\right)$; 7.03-7.89, (m, 15H, $\left.\mathrm{Ar}-\mathrm{H}\right) ; 8.58$, (s, $1 \mathrm{H}, \mathrm{N}-\mathrm{CH}=\mathrm{C}) ;{ }^{13} \mathrm{C} \mathrm{NMR}\left(150 \mathrm{MHz}, \mathrm{CDCl}_{3}\right): 66.4,115.0$ (2C), 122.2 , 123.0, 128.5 (2C), 128.7 (2C), 128.8 (2C), 129.6 (2C), 129.7 (2C), 130.6, 132.9, 133.6 (2C), 137.8, 156.4, 157.7, 167.1, 189.4; IR (KBr, $\mathrm{cm}^{-1}$ ): 3137, 2924, 1774, 1647, 1600, 1419; HRMS (ESI): $m / z\left[\mathrm{M}+\mathrm{H}^{+}\right]$calculated for monoisotopic mass 383.1317 , found 383.1390 .

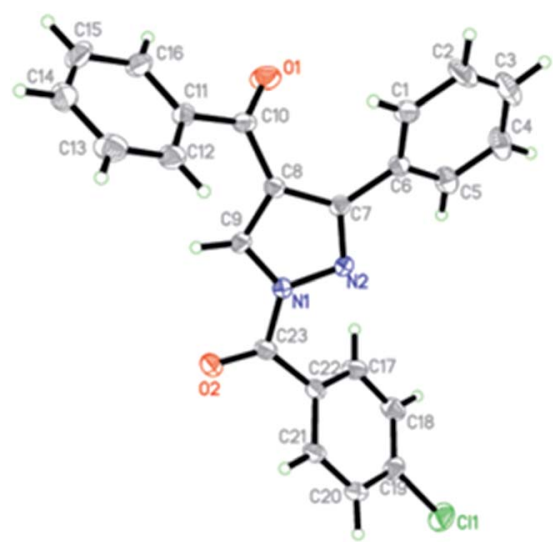

Fig. 2 Molecular structure of compound $5 f$. 
Table 1 The chlorophyll content of barnyard grass treated with target compound $\left(0.05 \mathrm{mmol} \mathrm{m}^{-2}\right)^{a}$

\begin{tabular}{|c|c|c|c|}
\hline Compound & $\mathrm{R}$ & Chlorophyll a content $\left(\mathrm{mg} \mathrm{g}^{-1}\right)$ & Chlorophyll b content $\left(\mathrm{mg} \mathrm{g}^{-1}\right)$ \\
\hline $5 a$ & & $130.44 \pm 1.64$ & $54.75 \pm 1.53$ \\
\hline $5 b$ & & $132.92 \pm 2.23$ & $57.72 \pm 0.56$ \\
\hline $5 c$ & & $133.01 \pm 2.43$ & $59.45 \pm 1.36$ \\
\hline $5 d$ & & $130.61 \pm 1.94$ & $55.32 \pm 0.28$ \\
\hline $5 e$ & & $132.78 \pm 2.97$ & $57.19 \pm 0.45$ \\
\hline $5 f$ & & $131.65 \pm 2.56$ & $56.61 \pm 1.09$ \\
\hline $5 g$ & & $90.78 \pm 2.34$ & $38.48 \pm 0.23$ \\
\hline $5 \mathbf{h}$ & & $115.53 \pm 2.36$ & $48.64 \pm 0.27$ \\
\hline $5 \mathbf{i}$ & & $132.89 \pm 2.23$ & $58.46 \pm 1.38$ \\
\hline $5 \mathbf{j}$ & & $132.19 \pm 1.57$ & $57.05 \pm 0.34$ \\
\hline $5 \mathbf{k}$ & $\mathrm{H}$ & $131.76 \pm 2.01$ & $54.29 \pm 0.79$ \\
\hline 51 & & $132.99 \pm 1.08$ & $58.97 \pm 1.19$ \\
\hline $5 \mathrm{~m}$ & & $133.13 \pm 1.34$ & $59.69 \pm 1.79$ \\
\hline $5 n$ & & $85.65 \pm 1.87$ & $34.49 \pm 0.94$ \\
\hline 50 & & $96.56 \pm 1.09$ & $40.32 \pm 1.31$ \\
\hline $5 p$ & & $124.15 \pm 2.09$ & $52.82 \pm 1.65$ \\
\hline $5 q$ & & $119.35 \pm 2.28$ & $50.12 \pm 0.97$ \\
\hline $5 \mathbf{r}$ & & $127.95 \pm 2.09$ & $53.62 \pm 1.05$ \\
\hline $5 s$ & $-\mathrm{CH}_{2} \mathrm{OCCH}_{3}$ & $113.61 \pm 1.26$ & $50.59 \pm 1.83$ \\
\hline
\end{tabular}


Table 1 (Contd.)

\begin{tabular}{|c|c|c|c|}
\hline Compound & $\mathrm{R}$ & Chlorophyll a content $\left(\mathrm{mg} \mathrm{g}^{-1}\right)$ & Chlorophyll b content $\left(\mathrm{mg} \mathrm{g}^{-1}\right)$ \\
\hline $5 t$ & $-\underset{\mathrm{O}}{\mathrm{O}}-\mathrm{Ph}$ & $121.18 \pm 1.83$ & $53.89 \pm 1.48$ \\
\hline CK & & $132.16 \pm 1.37$ & $59.72 \pm 0.94$ \\
\hline Pyrazoxyfen & & $128.29 \pm 1.28$ & $54.97 \pm 1.39$ \\
\hline
\end{tabular}

3-Phenyl-1-(2'-phenyl-5' -methyl-4' ${ }^{\prime}$-isoxazole)formacyl-pyrazole4-benzophenone (5p). White solid; yield: $83 \%$; mp: $178-180{ }^{\circ} \mathrm{C}$; ${ }^{1} \mathrm{H}$ NMR (600 MHz, $\left.\mathrm{CDCl}_{3}\right): 2.70,\left(\mathrm{~s}, 3 \mathrm{H},-\mathrm{CH}_{3}\right) ; 7.18-7.80,(\mathrm{~m}$, $15 \mathrm{H}, \mathrm{Ar}-\mathrm{H}) ; 8.59,(\mathrm{~s}, 1 \mathrm{H}, \mathrm{N}-\mathrm{CH}=\mathrm{C}) ;{ }^{13} \mathrm{C} \mathrm{NMR}\left(150 \mathrm{MHz}, \mathrm{CDCl}_{3}\right)$ : 13.2, 109.6, 123.0, 127.9 (2C), 128.2 (2C), 128.6 (2C), 128.7 (4C), 128.9, 129.4, 129.6 (2C), 130.1, 130.4, 133.4, 133.5, 137.7, 155.7, 161.2, 162.1, 175.5, 189.4; IR ( $\left.\mathrm{KBr}, \mathrm{cm}^{-1}\right): 3108,3078,1705$, 1666, 1597, 1416; HRMS (ESI): $m / z\left[\mathrm{M}+\mathrm{H}^{+}\right]$calculated for monoisotopic mass 434.1426, found 434.1503.

3-Phenyl-1-(5'-methyl-2'-(2-fluorine-5-chlorine)phenyl-4isoxazole)formacyl-pyrazole-4-benzophenone (5q). White solid; yield: 76\%; mp: 79-81 ${ }^{\circ} \mathrm{C} ;{ }^{1} \mathrm{H}$ NMR (600 $\left.\mathrm{MHz} \mathrm{CDCl}_{3}\right): 2.81$, (s, $\left.3 \mathrm{H},-\mathrm{CH}_{3}\right) ; 6.76-7.79,(\mathrm{~m}, 13 \mathrm{H}, \mathrm{Ar}-\mathrm{H}) ; 8.50,(\mathrm{~s}, 1 \mathrm{H}, \mathrm{N}-\mathrm{CH}=\mathrm{C})$; ${ }^{13} \mathrm{C}$ NMR (150 MHz, $\left.\mathrm{CDCl}_{3}\right): 13.5,111.4,114.2,114.4,117.8$, 117.9, 122.7, 125.3 (2C), 128.0 (2C), 128.6 (4C), 129.4, 129.6 (2C), 130.3, 131.2, 131.3, 133.5 (2C), 134.1 (2C), 137.8, 155.1, 159.7, 160.3, 161.4, 176.2, 189.5; IR ( $\left.\mathrm{KBr}, \mathrm{cm}^{-1}\right): 3135,3064,1717$, 1660, 1598, 1447; HRMS (ESI): $m / z\left[\mathrm{M}+\mathrm{H}^{+}\right]$calculated for monoisotopic mass 486.0942, found 486.1015.

3-Phenyl-1-(2'-trifluoromethyl-4'-methyl-3'-pyrazole)formacylpyrazole-4-benzophenone (5r). White solid; yield: $81 \%$; mp: 190 $192{ }^{\circ} \mathrm{C} ;{ }^{1} \mathrm{H}$ NMR $\left(600 \mathrm{MHz}, \mathrm{CDCl}_{3}\right)$ : 4.09, (s, 3H, $\left.-\mathrm{CH}_{3}\right) ; 7.43-7.91$, $(\mathrm{m}, 10 \mathrm{H}, \mathrm{Ar}-\mathrm{H}) ; 8.73(\mathrm{~s}, 1 \mathrm{H}, \mathrm{N}-\mathrm{CH}=\mathrm{C}) ; 8.94,(\mathrm{~s}, 1 \mathrm{H}, \mathrm{N}-\mathrm{CH}=\mathrm{C})$; ${ }^{13} \mathrm{C} \mathrm{NMR}\left(150 \mathrm{MHz}, \mathrm{CDCl}_{3}\right): 40.1,111.3,118.5,122.3,128.4(2 \mathrm{C})$, 128.7 (2C), 128.9 (2C), 129.6 (2C), 131.1, 133.5, 134.7, 137.9, 139.1, 144.5, 145.0, 156.1, 157.1, 189.4; IR (KBr, $\left.\mathrm{cm}^{-1}\right): 3162$, 3062, 1707, 1655, 1598, 1448; HRMS (ESI): $m / z\left[\mathrm{M}+\mathrm{H}^{+}\right]$calculated for monoisotopic mass 425.1147, found 425.1220.

3-Phenyl-1-acetyloxy-pyrazole-4-benzophenone (5s). Faint yellow powder; yield: $70 \%$; mp: $108-110{ }^{\circ} \mathrm{C} ;{ }^{1} \mathrm{H}$ NMR $(600 \mathrm{MHz}$, $\left.\mathrm{CDCl}_{3}\right): 2.26$, (s, 3H, $\left.-\mathrm{CH}_{3}\right) ; 5.56,\left(\mathrm{~s}, 2 \mathrm{H},-\mathrm{CH}_{2}-\right) ; 7.37-7.87,(\mathrm{~m}$, $10 \mathrm{H}, \mathrm{Ar}-\mathrm{H}) ; 8.51,(\mathrm{~s}, 1 \mathrm{H}, \mathrm{N}-\mathrm{CH}=\mathrm{C}) ;{ }^{13} \mathrm{C} \mathrm{NMR}\left(150 \mathrm{MHz}, \mathrm{CDCl}_{3}\right)$ : 20.5, 62.1, 123.0, 128.5 (2C), 128.7 (2C), 128.8 (2C), 129.6 (2C), 129.7, 130.6, 132.9, 133.6, 137.8, 156.3, 165.9, 170.4, 189.4; IR (KBr, $\mathrm{cm}^{-1}$ ): 3130, 2952, 1759, 1659, 1596, 1449; HRMS (ESI): $\mathrm{m} /$ $z\left[\mathrm{M}+\mathrm{H}^{+}\right]$calculated for monoisotopic mass 349.1110 , found 349.1187 .

3-Phenyl-1-benzenesulfonyl-pyrazole-4-benzophenone (5t). White solid; yield: $74 \%$; mp: $156-158{ }^{\circ} \mathrm{C}$; ${ }^{1} \mathrm{H}$ NMR $(600 \mathrm{MHz}$, $\left.\mathrm{CDCl}_{3}\right)$ : 7.33-8.14, (m, 15H, Ar-H); 8.43, (s, 1H, N-CH=C); ${ }^{13} \mathrm{C}$ NMR (150 MHz, $\mathrm{CDCl}_{3}$ ): 121.8, 128.3 (2C), 128.6 (2C), 128.7 (2C), 128.9 (2C), 129.4, 129.6 (2C), 129.7 (2C), 130.6, 133.5, 135.2, 135.5, 136.4, 137.8, 156.9, 189.3; IR ( $\left.\mathrm{KBr}, \mathrm{cm}^{-1}\right)$ : 3148, 3058,
1652, 1596, 1447; HRMS (ESI): $\mathrm{m} / z\left[\mathrm{M}+\mathrm{H}^{+}\right]$calculated for monoisotopic mass 389.0882 , found 389.0955 .

\section{X-ray diffraction}

The suitable single crystal of $\mathbf{5 f}$ was recrystallized from a mixture of ethyl acetate and petroleum ether (Fig. 2). The X-ray data were collected on a Rigaku RAXIS-RAPID diffractometer (Japan) with Mo-K $\alpha$ radiation $(\lambda=0.71073 \AA)$ at $293(2) \mathrm{K}$. A total of 5577 reflections were measured, of which 3266 independent reflections $\left(R_{\text {int }}=0.0382\right)$ were obtained in the range of $3.17^{\circ}<\theta$ $<25.00^{\circ}$ ( $h,-9$ to $9 ; k,-12$ to $10 ; l,-13$ to 11 ), and 1805 observed reflections with $I>2 \sigma(I)$ were used in refinement on $F^{2}$. The structure was solved by direct method using SHELXS-97 crystallographic software package. ${ }^{30}$ The full matrix least squares refinement based on $F^{2}$ gave final values of $R_{1}=0.1277$, $\omega R_{2}=0.3374$ and $\omega=1 /\left[\sigma^{2}\left(F_{0}{ }^{2}\right)+(0.0782 P)^{2}+9.9560 P\right]$, where $P$ $=\left({F_{0}}^{2}+2 F_{\mathrm{c}}{ }^{2}\right) / 3$ with $(\Delta / \sigma)_{\max }=0.983$ and $S=1.019$.

\section{Biological assays}

The greenhouse experiment was employed to measure the influences of target compounds to barnyard grass and maize. The concentration was set to $0.05 \mathrm{mmol} \mathrm{\textrm {m } ^ { - 2 }}$ after initial screening with pyrazoxyfen, and pyrazoxyfen was also used as the reference substance. After 15 days, all the compounds solutions were sprayed to the leaves and stems. Then 10 days later, chlorophyll content of barnyard grass was tested (Table 1).

Table 2 The chlorophyll content of maize treated with selected compounds $\left(1.25 \mathrm{mmol} \mathrm{m}^{-2}\right)^{a}$

\begin{tabular}{lrl}
\hline Compound & $\begin{array}{l}\text { Chlorophyll a content } \\
\left(\mathrm{mg} \mathrm{g}^{-1}\right)\end{array}$ & $\begin{array}{l}\text { Chlorophyll b } \\
\text { content }\left(\mathrm{mg} \mathrm{g}^{-1}\right)\end{array}$ \\
\hline $\mathbf{5 g}$ & $44.57 \pm 1.65$ & $15.19 \pm 0.09$ \\
$\mathbf{5 h}$ & $54.56 \pm 1.82$ & $17.92 \pm 0.27$ \\
$\mathbf{5 n}$ & $35.34 \pm 0.97$ & $12.72 \pm 0.69$ \\
$\mathbf{5 o}$ & $64.70 \pm 1.56$ & $20.49 \pm 1.06$ \\
$\mathbf{5 q}$ & $55.32 \pm 1.59$ & $17.63 \pm 1.12$ \\
$\mathbf{5 s}$ & $50.65 \pm 0.93$ & $16.02 \pm 0.58$ \\
$\mathbf{5 t}$ & $60.13 \pm 1.41$ & $18.63 \pm 0.83$ \\
CK & $109.73 \pm 1.27$ & $31.50 \pm 1.13$ \\
Pyrazoxyfen & $43.86 \pm 0.99$ & $12.94 \pm 0.88$ \\
& &
\end{tabular}

\footnotetext{
${ }^{a}$ Note: CK is for water treated.
} 
The safety to maize of the selected compounds was also determined. The pyrazoxyfen concentration was set to $1.25 \mathrm{mmol} \mathrm{m}^{-2}$ in order to make it injury to maize. The spraying treatment was conducted at the same dosage with pyrazoxyfen when the maize had reached the two-leaf stage. After 7 days, the chlorophyll content of the maize was determined (Table 2).

\section{Computational methods}

Compounds were constructed and optimized using SYBYL-X 2.0 and Gasteiger-Huckel charges were calculated for them. ${ }^{\mathbf{3 1}}$ The crystal structure of AtHPPD was obtained from the Protein Data Bank (PDB ID 1TFZ). Before docking, the protein structure was prepared in Accelrys Discovery Studio 2.5 (DS 2.5) to remove the water and some other co-crystallized small molecules. After the protein prepared, the active site of the protein was defined based on volume occupied by the known ligand pose already in an active site. During the docking process top 10 conformations were generated for each ligand based on docking score value after the energy minimization using the smart minimize method in DS 2.5.

\section{Results and discussion}

\section{Chemistry}

In this research, compound 4 was synthesized with 1,3diphenylpropane-1,3-dione, dimethylformamide dimethylacetal and $\mathrm{N}_{2} \mathrm{H}_{4} \cdot 2 \mathrm{HCl}$ as the starting materials, and dry toluene as solvent. It should be noted that the yield of compound 4 was promoted to $89.5 \%$ when reaction time prolonged to $1.5 \mathrm{~h}$ and appropriate distilled water added compared with the method of the ref. 26. It might due to that the $\mathrm{N}_{2} \mathrm{H}_{4} \cdot 2 \mathrm{HCl}$ dissolved adequately while adding distilled water.

The procedure to compounds $5 \mathbf{a}-\mathbf{t}$ was $N$-acylation reaction. The substitute structure affected the yields significantly due to the electronic effect. Compounds 5a-5d with benzoyl chloride or Me substituted benzoyl chloride used as acylated reagent were obtained in the high yield, such as the yield of compound 5b was increased to $90 \%$. While $p$-substituted phenyl with electron-withdrawing groups like $-\mathrm{Cl},-\mathrm{F},-\mathrm{CF}_{3}$ and $-\mathrm{NO}_{2}$, the yields were decreased largely, especially the yield of compound $\mathbf{5 i}$ was decrease to $30 \%$.

The structures of all the synthesized compounds were confirmed by ${ }^{1} \mathrm{H}$ NMR, ${ }^{13} \mathrm{C}$ NMR, and HRMS analyses. All target compounds showed similar spectroscopic characteristics due to the structural similarity. Single peak present at around 8.50$8.80 \mathrm{ppm}$ in ${ }^{1} \mathrm{H}$ NMR spectra correspond to the proton of pyrazole. The carbonyl connected to the 4 position of the pyrazole showed a shift at $189 \mathrm{ppm}$ in the ${ }^{13} \mathrm{C}$ NMR spectrum, and the shift around $167 \mathrm{ppm}$ correspond to carbonyls connected to $N$ substituted of the pyrazole.

\section{Structure analysis}

The molecular structure of $\mathbf{5 f}$ was shown in Fig. 2. Compound $\mathbf{5 f}$ contained three benzene rings and a pyrazole ring. The pyrazole ring and benzene ring are not coplanar with the dihedral angle being $46.726(279)^{\circ}$. In addition, the second benzene ring [C11, $\mathrm{C} 12, \mathrm{C} 13, \mathrm{C} 14, \mathrm{C} 15$, and $\mathrm{C} 16]$ is almost vertical to the first one $[\mathrm{C} 1, \mathrm{C} 2, \mathrm{C} 3, \mathrm{C} 4, \mathrm{C} 5$, and C6] with the dihedral angle being 74.64 $(2)^{\circ}$. No significant $\pi-\pi$ interactions were found in the crystal structure.

\section{Biological activity}

At present, HPPD inhibitory activity was evaluated through different assays. The enzyme assay in vitro is the most common method which tested by monitoring the production of maleylacetoacetate at $318 \mathrm{~nm} \cdot{ }^{17,18}$ However, the most pyrazole HPPD inhibitors could not directly inhibit the HPPD activity in vitro. In fact, pyrazoxyfen have to metabolized to 4-(2,4dichlorobenzoyl)-1,3-dimethyl-5-hydroxypyrazole in plant. The HPPD assay revealed that pyrazoxyfen inhibited the enzyme activity with the $\mathrm{IC}_{50}$ value of $7.5 \mu \mathrm{M}$ while the values of its metabolite is $13 \mathrm{nM}$. These data strongly suggest that the pyrazole herbicide inhibit HPPD after conversion to the herbicidally active metabolite in plants. ${ }^{32}$

The synthesized pyrazole benzophenone derivatives didn't inhibit the growth of barnyard grass or maize in the first 7 days, and then it showed typical bleaching injury symptoms to plants until the plants withered. The post-emergent herbicidal activities of compounds 5a-t were tested against barnyard grass. The commercial pyrazole herbicide pyrazoxyfen was selected as a positive control. As showed in Table 1, most of the compounds showed some extent herbicidal activities via decreasing the concentration of chlorophyll a and chlorophyll b, such as compounds $\mathbf{5 a - j}, \mathbf{5 l}-\mathbf{q}, \mathbf{5 s}, \mathbf{5 t}$. Most surprisingly, compounds 5g, 5h, 5n, 5o, 5q, 5s, 5t displayed the highest herbicidal activity which were superior to pyrazoxyfen.

Table 3 The calculated value of binding free energy of target compounds

\begin{tabular}{|c|c|c|c|c|c|}
\hline Compound & $\begin{array}{l}\text { Binding free energy } \\
\left(\mathrm{kJ} \mathrm{mol}^{-1}\right)\end{array}$ & Compound & $\begin{array}{l}\text { Binding free energy } \\
\left(\mathrm{kJ} \mathrm{mol}{ }^{-1}\right)\end{array}$ & Compound & $\begin{array}{l}\text { Binding free energy } \\
\left(\mathrm{kJ} \mathrm{mol}{ }^{-1}\right)\end{array}$ \\
\hline $5 \mathbf{a}$ & -7.723 & $5 h$ & -8.554 & 50 & -8.982 \\
\hline $5 b$ & -4.242 & $5 \mathbf{i}$ & -7.607 & $5 p$ & -7.495 \\
\hline $5 c$ & -6.008 & $5 \mathbf{j}$ & -9.309 & $5 q$ & -4.472 \\
\hline $5 d$ & -8.354 & $5 \mathbf{k}$ & -5.283 & $5 r$ & -4.538 \\
\hline $5 e$ & -5.654 & 51 & -9.180 & $5 s$ & -4.654 \\
\hline $5 f$ & -7.746 & $5 \mathrm{~m}$ & -2.312 & $5 t$ & -6.985 \\
\hline $5 g$ & -8.652 & $5 n$ & -9.236 & & \\
\hline
\end{tabular}




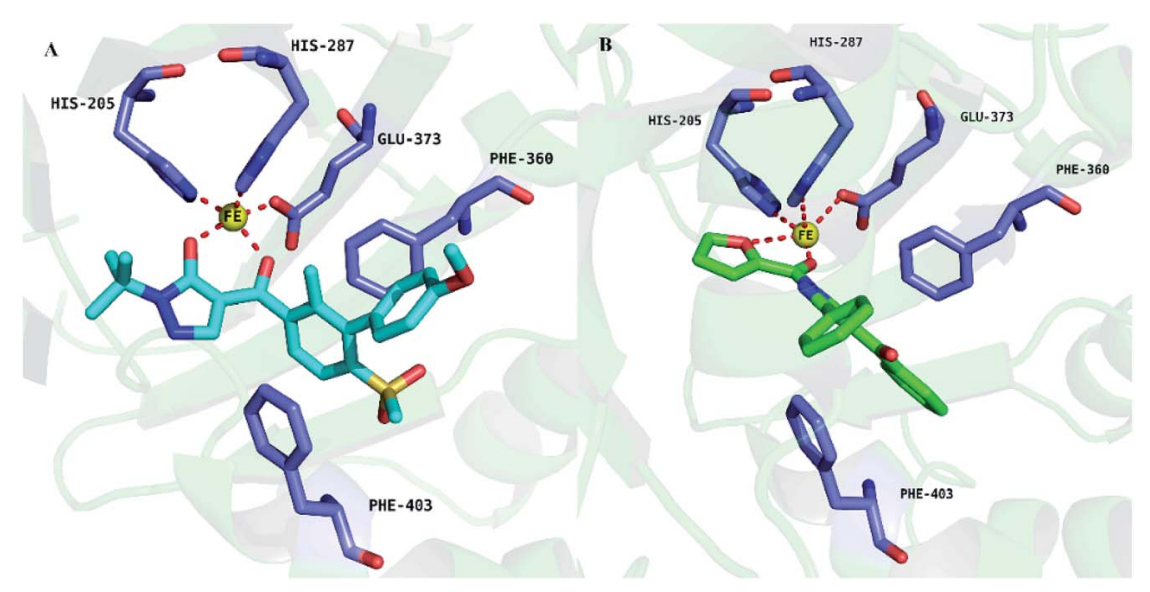

Fig. 3 Binding modes of ligand DAS869 (PDB ID: 1TFZ) (A) and compound $5 n$ with 1TFZ (B). (DAS869 is shown in cyan sticks and compound $5 n$ is shown in green sticks).

Comparing the activities of the compounds revealed that substituent $\mathrm{R}$ was the primary group which played a crucial role in the activity of the compounds. As can be seen from Table 1 , compounds $\mathbf{5 n}, \mathbf{5 q}$ and $\mathbf{5 p}$ with an aromatic fivemember ring at $\mathrm{R}$ displayed increasing herbicidal activity compared to compounds with benzoyl at $\mathrm{R}$. It is very interesting that compound $\mathbf{5 n}$ (furan) showed most strong herbicidal activity. Furthermore, compounds 5o, 5s and 5t with ethoxy substitution also found to have herbicidal activity against the barnyard grass. In contrast, compounds with benzoyl at $\mathrm{R}$ exhibited much lower herbicidal activity, even if compounds $\mathbf{5 g}$ and $\mathbf{5 h}$ showed more potent inhibition against barnyard grass, superior to pyrazoxyfen.

Compounds $5 \mathbf{g}, \mathbf{5 h}, \mathbf{5 n}, \mathbf{5 o}, \mathbf{5 q}, \mathbf{5 s}$, 5t were selected to determine the safety to maize after herbicidal test. As shown in Table 2, some compounds were safe compared with pyrazoxyfen. Compound $5 \mathbf{n}$ exhibited the best herbicidal activities against barnyard grass, unfortunately, it showed poor security to maize. Therefore, compound $\mathbf{5 0}$ may be the candidate as a herbicide for weed control in maize, and it was selected for further crop selectivity testing. The binding free energy of compound 5o with HPPD was relatively low, and that was agreed with the results of bioassay activity research (Table 3).

Compound $\mathbf{5 n}$ was selected to docking with AtHPPD for it showed the best herbicidal activity. The important interactions of $\mathbf{5 n}$ with the active site of AtHPPD were presented in Fig. 3. The bidentate coordination of the pyrazoxyfen part with $\mathrm{Fe}^{2+}$ and the sandwiched $\pi-\pi$ interaction of the benzene ring with Phe403 and Phe360 were mainly two interactions of AtHPPD with inhibitor. Compound $\mathbf{5 n}$ bind to HPPD as the same configuration as the DAS869. The oxygen of furan and carbonyl were responsible for forming a bidentate coordination with the $\mathrm{Fe}^{2+}$. Meanwhile, benzene ring which links to 4-position of pyrazole formed a favorable sandwich $\pi-\pi$ interaction with residues Phe360 and Phe403 increasing hydrophobic interaction with amino acid of AtHPPD in the active pocket. The result also indicated that compound $\mathbf{5 n}$ might be a promising herbicide candidate.

\section{Conclusion}

In conclusion, a series of novel pyrazole derivatives were designed and identified as potent HPPD inhibitors. Most of the synthesized compounds displayed excellent herbicidal activities, some of them even superior to the commercial herbicide pyrazoxyfen. Much to our delight, compounds 5g, 5h, 5n, 5o, 5p, $\mathbf{5 q}, \mathbf{5 s}, \mathbf{5 t}$ displayed promising herbicidal activities at a rate of $0.05 \mathrm{mmol} \mathrm{m}^{-2}$. It is inspired that compounds 50 showed better safety to maize. Therefore, the herbicidal activity of compound 50 was better than pyrazoxyfen. Besides, it was safe when used on maize. These results indicated that pyrazole derivatives could be novel lead compounds for novel herbicide discovery.

\section{Conflicts of interest}

The authors have no conflicts of interest to declare.

\section{Acknowledgements}

We are very grateful to the National Natural Science Foundation of China (31572042), the Natural Science Foundation of Heilongjiang Province (ZD2017002), and the Research Science Foundation in Technology Innovation of Harbin (2015RAYXJ010).

\section{References}

1 D. L. Lee, M. P. Prisbylla, T. H. Cromartie, D. P. Dagarin, S. W. Howard, W. M. Provan, M. K. Ellis, T. Fraser and L. C. Mutter, Weed Sci., 1997, 45, 601-609.

2 G. R. Moran, Arch. Biochem. Biophys., 2005, 433, 117-128.

3 D. W. Wang, H. Y. Lin, R. J. Cao, Z. Z. Ming, T. Chen, G. F. Hao, W. C. Yang and G. F. Yang, Pest Manage. Sci., 2015, 71, 1122-1132.

4 C. Raspail, M. Graindorge, Y. Moreau, S. Crouzy, B. Lefebvre, A. Y. Robin, R. Dumas and M. Matringe, J. Biol. Chem., 2011, 286, 26061-26070. 
5 A. Santucci, G. Bernardini, D. Braconi, E. Petricci and F. Manetti, J. Med. Chem., 2017, 60, 4101-4125.

6 S. R. Norris, T. R. Barrette and D. DellaPenna, Plant Cell, 1995, 7, 2139-2149.

7 H. Maeda and D. DellaPenna, Curr. Opin. Plant Biol., 2007, 10, 260-265.

8 F. E. Dayan, S. O. Duke, A. Sauldubois, N. Singh, C. McCurdy and C. Cantrell, Phytochemistry, 2007, 68, 2004-2014.

9 A. S. Godar, V. K. Varanasi, S. Nakka, P. V. V. Prasad, C. R. Thompson and J. Mithila, PLoS One, 2015, 10, 1-17.

10 A. J. Jhala, L. D. Sandell, N. Rana, G. R. Kruger and S. Z. Knezevic, Weed Technol., 2014, 28, 28-38.

11 M. J. Walsh, K. Stratford, K. Stone and S. B. Powles, Weed Technol., 2012, 26, 341-347.

12 J. D. Bollman, C. M. Boerboom, R. L. Becker and V. A. Fritz, Weed Technol., 2008, 22, 666-674.

13 M. M. Williams and J. K. Pataky, Weed Sci., 2010, 58, 289294.

14 S. Lindstedt, E. Holme, E. A. Lock, O. Hjalmarson and B. Strandvik, Lancet, 1992, 340, 813-817.

15 A. J. Woodyard, G. A. Bollero and D. E. Riechers, Weed Technol., 2009, 23, 513-518.

16 M. Baalouch, A. De Mesmaeker and R. Beaudegnies, Tetrahedron Lett., 2013, 54, 557-561.

17 D. W. Wang, H. Y. Lin, R. J. Cao, T. Chen, F. X. Wu, G. F. Hao, Q. Chen, W. C. Yang and G. F. Yang, J. Agric. Food Chem., 2015, 63, 5587-5596.

18 D. W. Wang, H. Y. Lin, B. He, F. X. Wu, T. Chen, Q. Chen, W. C. Yang and G. F. Yang, J. Agric. Food Chem., 2016, 64, 8986-8993.
19 C. D. Duarte, E. J. Barreiro and C. A. M. Fraga, Mini-Rev. Med. Chem., 2007, 7, 1108-1119.

20 A. Y. Shaw, H. H. Liau, P. J. Lu, C. N. Yang, C. H. Lee, J. Y. Chen, Z. G. Xu and G. Flynn, Bioorg. Med. Chem., 2010, 18, 3270-3278.

21 Y. L. Xu, H. Y. Lin, X. Ruan, S. G. Yang, G. F. Hao, W. C. Yang and G. F. Yang, Eur. J. Med. Chem., 2015, 92, 427-438.

22 Y. L. Xu, H. Y. Lin, R. J. Cao, Z. Z. Ming, W. C. Yang and G. F. Yang, Bioorg. Med. Chem., 2014, 22, 5194-5211.

23 F. Ye, S. L. Wu, L. X. Zhao, H. T. Qu, S. Gao and Y. Fu, Heterocycles, 2015, 91, 1256-1268.

24 Y. Fu, Y. N. Sun, K. H. Yi, M. Q. Li, H. F. Cao, J. Z. Li and F. Ye, Molecules, 2017, 22, 959.

25 Y. Fu, Y. N. Sun, H. F. Cao, K. H. Yi, X. Z. Li, J. Z. Li and F. Ye, Comb. Chem. High Throughput Screening, 2017, 20, DOI: 10.2174/1386207320666170622073738.

26 M. A. Al-Shiekh, Org. Prep. Proced. Int., 2005, 37, 223-230.

27 G. Menozzi, L. Merello, P. Fossa, S. Schenone, A. Ranise, L. Mosti, F. Bondavalli, R. Loddo, C. Murgioni and V. Mascia, Bioorg. Med. Chem., 2004, 12, 5465-5483.

28 P. Schenone, L. Mosti and G. Menozzi, J. Heterocycl. Chem., 1982, 19, 1355-1361.

29 D. J. Hogenkamp, T. B. C. Johenstion and K. W. Gee, WO Pat., 2005/108347, 2005.

30 G. M. Sheldrick, Acta Crystallogr., Sect. A: Found. Crystallogr., 2008, 64, 112-122.

31 SYBYL, Version 6.9, Tripos.

32 H. Matsumoto, ACS Symp. Ser., 2005, 892, 161-171. 